\title{
DETERMINACIÓN DE INSECTICIDAS ORGANOFOSFORADOS EN NOPAL FRESCO Y DESHIDRATADO
}

\section{DETERMINATION OF ORGANOPHOSPHATE INSECTICIDES IN FRESH AND DRIED PRICKLY PEAR CACTUS PADS}

\section{María Lourdes Aldana Madrid ${ }^{1 *}$, María del Carmen García Moraga ${ }^{1}$, Guillermo Rodríguez Olibarria ${ }^{1}$, María Isabel Silveira Gramont ${ }^{1}$ y Ana Isabel Valenzuela Quintanar ${ }^{2}$}

\footnotetext{
${ }^{1}$ Depto. de Investigación y Posgrado en Alimentos, Universidad de Sonora. Rosales y Transversal s/n, Centro. 83000, Hermosillo, Sonora, México. Tel. y Fax (662) 25922 07, 08 y 09. ${ }^{2}$ Centro de Investigación en Alimentación y Desarrollo, A. C. Carr. A la Victoria km. 0.6. 83000, Hermosillo, Sonora, México.

* Autor para correspondencia (laldana@guayacan.uson.mx)
}

\section{RESUMEN}

El este estudio se identificaron y cuantificaron los insecticidas organofosforados presentes en cladodios de nopal (Opuntia ficus indica) fresco y deshidratado, mediante extracción con el método de fasesólida dispersiva, e identificación y cuantificación por cromatografía de gases. Se muestrearon seis campos de nopal cercanos a Hermosillo, Sonora, México, y se llevó a cabo una encuesta para conocer los antecedentes del predio referentes al tipo y frecuencia de aplicación del insecticida, así como área, número de plantas, fecha de plantación y cosecha. Los insecticidas organofosforados analizados fueron malatión, paratión metílico, diazinón y clorpirifos. Los porcentajes de recuperación del método de extracción fueron de 90 a $96 \%$, con una variación de $12 \%$. En todas las muestras de nopal fresco $(n=24), 60$ $\%$ contenía tres de los insecticidas analizados, y en el resto hubo los cuatro insecticidas. El malatión se detectó en $97 \%$ de las muestras, pero siempre por debajo de los límites máximos de residuos (LMRs) para hortalizas. Además se detectaron residuos de dos insecticidas no autorizados para su uso en hortalizas (clorpirifos y paratión metílico). El nopal deshidratado no presentó residuos de insecticidas. En las muestras de nopal fresco las concentraciones de insecticidas fueron menores al valor de la ingesta diaria admisible, para malatión, paratión metílico, diazinón y clorpirifos. El potencial de riesgo toxicológico proveniente del consumo de nopal fresco y su producto deshidratado es mínimo, ya que la ingesta diaria estimada fue 1000 veces menor que la dosis admisible.

Palabras clave: Opuntia ficus indica, ingesta diaria admisible, ingesta diaria estimada, potencial de riesgo toxicológico.

\section{SUMMARY}

The objective of this study was to identify and quantify the organophosphate insecticides in fresh and dried prickly pear pads (Opuntia ficus indica), by using the dispersive solid-phase extraction method followed by gas chromatography. Sampling was carried out at six prickly pear orchards near Hermosillo city, Sonora, México. A survey was done to know the field management regarding to the application frequency of the different insecticides, the total area sprayed, number of plant treated with insecticides, as well as planting and harvesting dates. The organophosphate insecticides analyzed were: malathion, methyl parathion, diazinon and chlorpyrifos. The calculated recovery percentage of the extraction protocol ranged from 90 to $96 \%$ with a coefficient of variation of $12 \%$. Out of the 24 samples evaluated, $60 \%$ contained three of the insecticides, whereas in the remaining samples all four insecticides were found. Malathion was found in $97 \%$ of the samples although its concentration was within the maximum residue limits (MRLs) for vegetables. Additionally, two insecticide residues not authorized for vegetables (chlorpyrifos and methyl parathion) were detected. No insecticide residues were detected in dehydrated prickly pear pads. In samples of fresh prickly pear pads, the concentrations of malathion, methyl parathion, diazinon and chlorpyrifos were found to be below the acceptable daily intake level. It is concluded that the potential toxicological risk related to the consumption of fresh prickly pear pads and its dehydrated product is minimal because the estimated daily ingestion is $\mathbf{1 0 0 0}$ times lower than the admissible dose.

Index words: Opuntia ficus indica, acceptable daily intake, estimated daily ingestion, potential toxicological risk.

\section{INTRODUCCIÓN}

Los insecticidas organofosforados tienen en la actualidad un papel preponderante como plaguicidas fitosanitarios que benefician la producción y calidad de frutas y hortalizas. Sin embargo, cuando no se aplican correctamente pueden dispersarse en el ambiente y representar un peligro de salud pública (Ripley et al., 2001). Los gobiernos y las organizaciones internacionales han establecido una serie de límites máximos de residuos (LMRs) que son bastante restrictivos en muchos casos e incluso por debajo de los recomendados por la Organización de las Naciones Unidas para la Agricultura y la Alimentación y la Organización Mundial de la Salud (Torres et al., 1996; Dogheim et al., 1996 y 1999; European Community, Brussels, 1990), para controlar los residuos de insecticidas que resultan de su empleo autorizado en alimentos. El control de calidad se debería cumplir para la comercialización de productos 
agrícolas, tanto en el ámbito nacional como internacional, y su cumplimiento estricto garantizaría que los productos agrícolas lleguen al mercado con niveles de residuos de insecticidas por debajo del LMRs (Coscolla, 1993; Juran y Gryma, 1997). La verificación continua de residuos de plaguicidas en alimentos, para determinar el grado de exposición de una población humana, es importante para conocer el grado de contaminación y las posibles consecuencias toxicológicas a largo plazo (Dogheim et al., $1996 \mathrm{y}$ 1999), tanto de moléculas de nueva síntesis como de las que han sido restringidas o prohibidas (Navarro et al., 1992; Haney y Harris, 1999).

El uso de estos compuestos en el cultivo del nopal (Opuntia ficus indica) no es la excepción, dado que estos productos se consumen crudos (en ensaladas, como fuente de fibra y otros nutrientes) y en algunas ocasiones procesados (deshidratado, para la elaboración de cosméticos) (CIESTAAM-ASERCA, 1995), por lo que es necesario conocer el riesgo que representa su consumo, ya sea en forma natural o procesado. En la norma del Codex para nopal (Codex Stan 185,1993) se exponen las disposiciones relativas a calidad, y presentación, así como de marcado o etiquetado e higiene del producto; sin embargo, no establece ni especifica la concentración permisible de contaminantes, tales como residuos de plaguicidas. Estas normas sólo están reconocidas para hortalizas en general y en algunas de ellas cuando son de uso frecuente.

En México la producción de nopal verdura ocupa el sexto lugar en volumen de producción de hortalizas y el octavo lugar en valor de la producción (Berger et al., 2006). En el Estado de Sonora la producción de cladodios tiernos de nopal se ubica en el 11 lugar a nivel nacional (con 328.3 t), y en el lugar 14 en superficie sembrada (SIAP-SAGARPA, 2007). En Sonora se ha incrementado su consumo de manera considerable (60 \%) (Díaz, Com. personal $)^{1}$, con la consecuente inquietud de un grupo de productores por conocer los residuos presentes de insecticidas en la penca y en el producto procesado.

Entre los métodos de análisis de multirresiduos de insecticidas organofosforados, la cromatografía de gases se considera un método adecuado, por lo que en este caso la extracción de tales insecticidas se hizo mediante la técnica en fase-sólida dispersiva (FSD) que se caracteriza por ser rápida, sensible y por emplear un volumen reducido de disolvente y de muestra, que la hacen ser económica y de baja contaminación ambiental (Anastassiades et al., 2003). El objetivo del presente estudio fue determinar la presencia de insecticidas organofosforados: malatión, paratión metí-

\footnotetext{
${ }^{1}$ Díaz Mireya (2007). Química Bióloga. Líder de la Asociación de Productores de Nopal en el Estado de Sonora.
}

lico, diazinón y clorpirifos, en nopal fresco cultivado en campos agrícolas de la localidad, así como en nopal deshidratado. También se calculó la ingesta diaria estimada (IDE) de nopal, con el fin de determinar el margen de seguridad de aquellos residuos que pudieran estar presentes en nopal.

\section{MATERIALES Y MÉTODOS}

Criterios de selección de los campos muestreados. Se consideró que los campos seleccionados abastecieran a las empresas comercializadoras de Hermosillo, Sonora, México, y que éstas suministran de nopal a sus habitantes. Los campos elegidos fueron: dos de la Región GuaymasEmpalme (km 104-105), dos de la Costa de Hermosillo

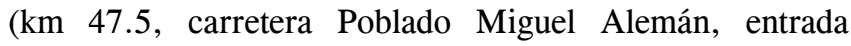
Puerto Libertad), y dos situados a la salida Noroeste de la ciudad (carretera Hermosillo-Ures, km 3 y km 12) (Figura $1)$.

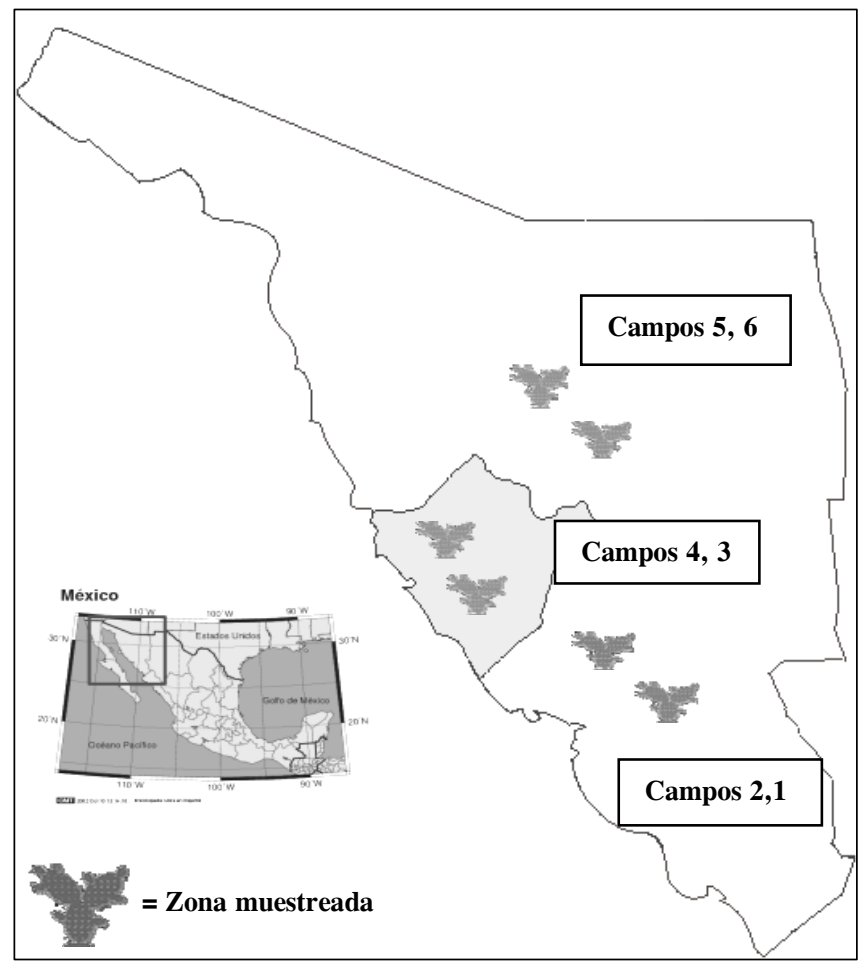

Figura 1. Ubicación de las Localidades del Estado de Sonora, México, en las que se hicieron los muestreos del nopal.

En Guaymas (Campo 1) la plantación seleccionada para tomar las muestras de nopal tenía una edad aproximada de tres años. En Empalme (Campo 2) la parcela limita por los cuatro puntos cardinales con terreno sin cultivar, y la edad aproximada era de cinco años. Los cultivos visitados en la Costa de Hermosillo (Campos 3 y 4) colindan por sus cuatro lados con terrenos sin cultivar, y las plantaciones eran de más de cinco años. El Campo 5 ubicado en la región 
noreste (salida a Hermosillo), colinda al Norte y al Este con terrenos sin cultivar, al Sur con una casa habitación y al Oeste con un cultivo de hortaliza de temporada, generalmente cebolla (Allium cepa L.); las plantas tenían más de cinco años. La última parcela visitada (Campo 6) era de dos años y limita en sus cuatro lados con terrenos sin cultivar. En los predios muestreados se produce la variedad de nopal Copena y utilizan sistema de riego por goteo.

Encuesta a productores de nopal. Se aplicó una encuesta a productores de nopal, para obtener información del cultivo, como: fechas de plantación, número de surcos, cultivos colindantes, fechas de cosecha, última fecha de corte, uso de insecticidas, y última fecha de aplicación, entre otras.

Muestreo. Se analizó una población de 24 muestras de nopal fresco y 24 muestras de nopal deshidratado provenientes de seis plantaciones de nopal, las cuales fueron recolectadas manualmente de acuerdo con las especificaciones de muestreo indicadas en el Manual de Entrenamiento para Laboratorio de Pesticidas de la AOAC (Meloan, 1998).

Diseño del muestreo. Para obtener una muestra representativa y homogénea de nopal se sobrepuso una cuadrícula imaginaria en cada uno de los seis campos, y cada campo se dividió en nueve áreas. De cada área se recolectaron dos o tres porciones de $0.5 \mathrm{~kg}$, en 14 puntos de muestreo. Las muestras obtenidas se combinaron en función al punto cardinal al que pertenecían (norte, sur, este u oeste) para formar así cuatro muestras de cada campo. Finalmente, las muestras se trasladaron al laboratorio para su almacenamiento a $-20{ }^{\circ} \mathrm{C}$ y análisis posterior.

Selección de insecticidas. La selección de los insecticidas a estudiar, se apoyó en una revisión de trabajos realizados localmente sobre la detección de plaguicidas en líquidos corporales (Aldana et al., 2003) y en alimentos como hortalizas (Valenzuela et al., 2006), carnes (Vázquez et al., 1999), granos (Valdez et al., 2002; Vargas et al., 2002), camarón, y en matrices como sedimento y agua de granjas acuícolas (Burgos et al., 2005 y 2006).

En la oficina de Sanidad Vegetal se obtuvo información de los plaguicidas más utilizados en el Estado de Sonora, y se consideraron los resultados de las entrevistas aplicadas a productores que participaron en el estudio, y a otros productores vecinos. De esta investigación, los insecticidas organofosforados, junto con los carbamatos y piretroides, resultaron ser ampliamente utilizados. Se construyó así una lista de cuatro insecticidas organofosforados: malatión, paratión metílico, diazinón y clorpirifos.
Preparación de estándares. Se emplearon estándares certificados de malatión, paratión metílico, diazinón y clorpirifos (con 98.5, 99.7, 98.5 y $99.6 \%$ de pureza, respectivamente) de la marca Riedel-de Haën (RdH Laborchemikalien GmbH and Co KG D30926 Seelze). Para su preparación se consideraron los LMRs de hortalizas como tomate (Lycopersicum esculentum), calabaza (Cucurbita pepo L.) y chile (Capsicum annuum), que proporciona el Departamento de Agricultura de Estados Unidos (USDA, 1991), así como los de la Comisión Intersecretarial para el Control del Proceso y Uso de Plaguicidas, Fertilizantes y Sustancias Tóxicas (CICOPLAFEST, 1998) y del Codex Alimentarius (1999). Se elaboraron soluciones madre de cada estándar, con el procedimiento de Anastassiades et al. (2003), y a partir de ellas se hicieron una serie de diluciones en hexano (grado plaguicida) para obtener las concentraciones de $0.01,0.05,0.1,0.2,0.3, y 0.5 \mathrm{mg} \mathrm{kg}^{-1}$ que fueron utilizadas en la curva de calibración, lo cual se hizo por triplicado, y se fijaron en función de los límites de detección del equipo.

Preparación de la muestra. A partir de dos submuestras de $1.5 \mathrm{~kg}$ de cladodios (pencas) de nopal representativas de las nueve áreas muestreadas en cada campo, se procedió a efectuar el análisis de plaguicidas en nopal fresco y en nopal deshidratado.

Para la deshidratación del nopal se empleó un secador tipo túnel planta piloto (sin marca), a una temperatura de $70{ }^{\circ} \mathrm{C}$, hasta lograr una humedad final de $6 \%$ (Platt et $a l .$, 2003). Las pencas secas se quebraron y trituraron en un molino manual Azteca, luego se molieron en un molino Laboratory Mill 3100, para obtener un tamaño de partícula de 60 a 80 mesh, que facilita la extracción y cuantificación de los insecticidas.

La submuestra (en fresco) se homogeneizó en un biohomogeneizador Biospec M173/(28)-0 de dos velocidades, hasta tener una muestra lo más finamente molida. Las muestras se manejaron individualmente y se mantuvieron almacenadas a $-20{ }^{\circ} \mathrm{C}$.

Procedimiento de extracción. Para remover los residuos de plaguicidas del nopal, la extracción se hizo por fase sólida dispersiva, método propuesto por Anastassiades et al. (2003), y las muestras se trabajaron por triplicado. Una vez obtenido el extracto se inyectaron $3 \mu \mathrm{L}$ al cromatógrafo de gases. Con base en el área bajo la curva del cromatograma y por comparación de los tiempos de retención, se determinó la presencia o ausencia del plaguicida. Se emplearon tres controles para la muestra: blanco de muestra, blanco de muestra fortificado con los cuatro insecticidas a analizar y el blanco reactivo. 
Equipo. Se utilizó un cromatógrafo de gases Varian CP-3800 provisto con detector de captura de electrones y programa Star Chromatography Workstation, versión 5.51, provisto con una columna capilar DB 1701 de 0.25 $\mathrm{mm}$ de diámetro interno y $30 \mathrm{~m}$ de longitud, específica para insecticidas. Se utilizó como gas acarreador helio UAP, con un flujo de $2 \mathrm{ml} \mathrm{min}^{-1}$, y nitrógeno como gas de relle-

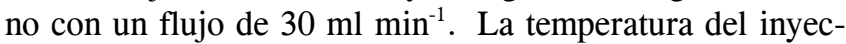
tor fue de $250{ }^{\circ} \mathrm{C}$ y la del detector de $300{ }^{\circ} \mathrm{C}$; la temperatura inicial de la columna fue de $120{ }^{\circ} \mathrm{C}$, que con un programa de cambios de temperatura se incrementó en $9{ }^{\circ} \mathrm{C}$ $\min ^{-1}$, hasta llegar a $280{ }^{\circ} \mathrm{C}$.

Aseguramiento de la calidad de los datos. En todos los análisis realizados durante la optimización y el análisis de residuos de plaguicidas organofosforados, se incluyó una muestra blanco o control negativo (tejido libre de plaguicidas), una muestra adicionada o control positivo (tejido blanco adicionado con la solución de trabajo a $100 \%$ de los LMR mencionados), un blanco reactivo y la solución de trabajo a $100 \%$ de los LMR.

Análisis estadístico. Los datos obtenidos de la optimización del método y del análisis de residuos fueron analizados mediante estadística descriptiva, con base en la media, desviación estándar, coeficiente de variación y coeficiente de correlación de las curvas de calibración de cada plaguicida.

Evaluación de riesgo toxicológico. Para determinar el grado de exposición es necesario conocer la composición de la dieta alimenticia media en Hermosillo, Sonora. Debido a que no se dispone de datos respecto a la proporción de nopal consumido en la región, se consideró el consumo per capita en la República Mexicana de $5.78 \mathrm{~kg}$ al año, reportado por Berger et al. (2006). La siguiente ecuación proporciona la ingesta diaria estimada (IDE), en mg de insecticida por kilogramo de peso corporal por día, que se compara con la ingesta diaria admisible (IDA) de iguales unidades, establecida para cada uno de los insecticidas:

$$
\mathrm{IDE}=\sum \mathrm{c}_{\mathrm{i}}\left(\frac{\mathrm{C}}{\mathrm{NDK}}\right)
$$

Donde: la $\sum \mathrm{c}_{\mathrm{i}}$ es la suma de las concentraciones en muestras de nopal $\left(\mathrm{mg} \mathrm{kg}^{-1}\right)$; $\mathrm{C}$ es el consumo de nopal anual por persona ( $\mathrm{kg}$ de nopal por persona al año); $\mathrm{N}$ es el total de muestras analizadas en cada campo, D es el número de días en un año, y $\mathrm{K}$ peso en $\mathrm{kg}$ por persona (Valenzuela et al., 2001). Para los cálculos se consideraron 70 $\mathrm{kg}$ de peso corporal en una persona promedio (Louvar y Louvar, 1998).

\section{RESULTADOS Y DISCUSIÓN}

De acuerdo con a las encuestas aplicadas a los responsables de los seis campos muestreados, solamente dos de ellos manifestaron haber utilizado plaguicidas y uno haber aplicado malatión.

Los porcentajes de recuperación obtenidos mediante el método utilizado fluctuaron en el intervalo de 90 a $96 \%$ en el nopal deshidratado y de 92 a $100 \%$ en nopal fresco, con coeficientes de variación $\leq 12 \%$ (Cuadro 1), que están dentro de los límites establecidos por USDA (1991) y los reportados por Anastassiades et al. (2003) para diazinón.

Cuadro 1. Porcentajes de recuperación y coeficientes de variación de plaguicidas organofosforados en nopal mediante el método optimizado de extracción en fase dispersiva.

\begin{tabular}{lccccc}
\hline \multicolumn{5}{l}{ Nopal deshidratado } & Nopal fresco \\
\cline { 2 - 6 } Insecticidas & $\begin{array}{c}\text { Recuperación } \\
(\%)\end{array}$ & $\begin{array}{c}\mathrm{CV} \\
(\%)\end{array}$ & $\begin{array}{c}\text { Recuperación } \\
(\%)\end{array}$ & $\begin{array}{c}\mathrm{CV} \\
(\%)\end{array}$ & $\begin{array}{c}\text { Valores* } \\
\text { aceptables } \\
(\%)\end{array}$ \\
\hline Diazinón & 96 & 7 & 100 & 5 & $60-110$ \\
Clorpirifos & 91 & 9 & 100 & 8 & $80-110$ \\
Malatión & 92 & 12 & 92 & 11 & $70-110$ \\
$\begin{array}{l}\text { Paratión } \\
\text { metílico }\end{array}$ & 90 & 11 & 96 & 9 & $65-110$ \\
\hline CV $=$ Coeficiente de variación $(\mathrm{n}=3)$ Referencia * & & & & & \\
\hline
\end{tabular}

En un cromatograma característico de la mezcla de insecticidas organofosforados obtenidos en este estudio (Figura 2), se observa buena separación de los analitos, con un tiempo de análisis de $17.5 \mathrm{~min}$. El coeficiente de correlación obtenido fue 0.98 para diazinón y paratión metílico, 0.91 para malatión y de 0.89 para clorpirifos.

En el nopal deshidratado no se detectaron los insecticidas de interés, en ninguno de los campos participantes, posiblemente debido a las propiedades físicas de los insecticidas organofosforados como son su solubilidad y volatilización (Coscolla, 1993).

Del total de muestras analizadas de nopal fresco, $60 \%$ contenía residuos de tres insecticidas, mientras que $40 \%$ restante mostró residuos de cuatro insecticidas. Particularmente en los Campos 1, 2 y 3 no se detectó presencia de insecticida alguno. Esto sugiere que probablemente las parcelas no fueron sometidas a aplicación reciente de insecticidas, o bien que las concentraciones se encontraban por debajo de las detectadas por el método de análisis realizado (10 $\mathrm{ng} \mathrm{mL}^{-1}$ para clorpirifos, malatión y paratión metílico, ó $100 \mathrm{ng} \mathrm{mL}^{-1}$ para diazinón). La cantidad de 
insecticida que permanece después de una aplicación está determinada por la naturaleza del insecticida (dosis, polaridad, características de aplicación) y por la configuración química de la superficie del vegetal. También pudieron influir las condiciones climatológicas (Coscolla, 1993; Reigart y James, 1999), como los vientos fuertes y lluvias esporádicas observadas antes de las fechas de muestreo. En los Campos 4, 5 y 6, en 97 \% de las muestras se identificó un pico en los cromatogramas que coincide con el de malatión, tanto en campos que aplican insecticida como en los que no aplican, lo que indica que aún no se había degradado, ya sea por mecanismos fisicoquímicos o por biodegradación.

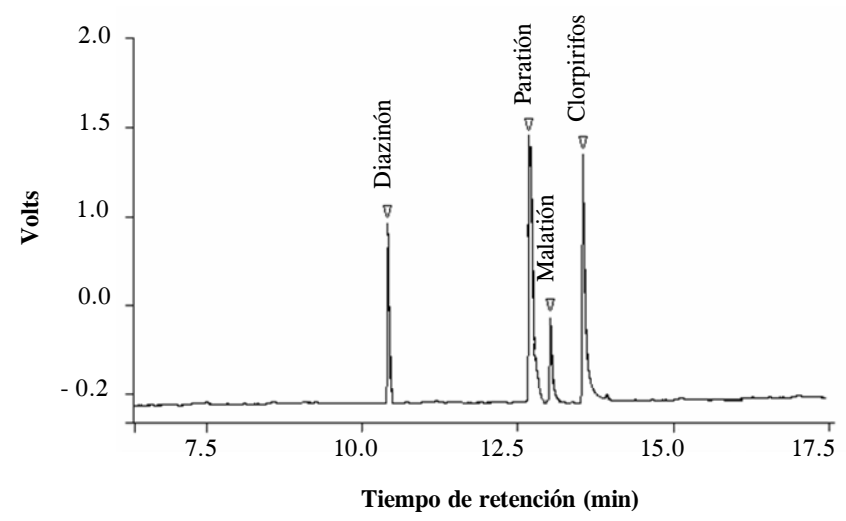

Figura 2. Cromatograma de la determinación de los insecticidas organofosforados. Tiempos de Retención; Diazinón, $t_{R}=54 \mathrm{~min}$; Clorpirifos, $t_{R}=12.79$ min; Malatión, $t_{R}=3.12$ min; Paratión Metílico, $t_{R}=$ 13.6 min.

Por las características de persistencia del insecticida, es posible que haya sido aplicado en el nopal o en campos agrícolas cercanos durante esos meses. A pesar de que el malatión fue detectado por debajo de los LMRs (para tomate y chile; Cuadro 2), debe considerarse que la ruptura de la molécula de malatión no implica el cese de sus efectos tóxicos, dado que el proceso pudiera generar metabolitos incluso más peligrosos y persistentes. En un experimento dirigido por Barlas en 1996 en la Universidad de Turquía y citado en el informe de Montenegro (2001), se observó que $10 \mathrm{~d}$ después de aplicar malatión al suelo, este insecticida organofosforado se había reducido a una cuarta parte del contenido original, pero también se habían formado otros metabolitos como ácido monocarboxílico (14 \%) y malaoxón (8 \%).

El riesgo al que se expone el consumidor de nopal fresco es difícil de evaluar porque no se dispone de información específica de este producto. Pero al comparar con las LMRs para otras hortalizas de uso frecuente (tomate, calabaza y chile), con los promedios obtenidos de las muestras positivas (Cuadro 2), se pudo observar que las muestras del Campo 4 contenían residuos de malatión comparables con los LMRs para tomate y chile, sin exceder los límites permisibles (para calabaza, el malatión no se encuentra en el listado de insecticidas aprobados por el Codex Alimentarius, 1999).

Cuadro 2. Comparación de la concentración de insecticidas encontrados en nopal y límites máximos de residuos (LMR) de otras hortalizas.

\begin{tabular}{|c|c|c|c|c|c|}
\hline \multirow[t]{2}{*}{$\mathrm{Campo}^{\dagger}$} & \multirow{2}{*}{$\begin{array}{l}\text { Insecticidas } \\
\text { encontrados } \\
\text { en nopal }\end{array}$} & \multirow{2}{*}{$\begin{array}{l}\text { Concentración } \\
\text { Promedio } \pm \mathrm{d} \text { e } \\
\left(\mathrm{mg} \mathrm{kg}^{-1}\right),\end{array}$} & \multicolumn{3}{|c|}{$\begin{array}{l}\mathrm{LMR}^{\dagger \dagger \dagger} \\
\left(\mathrm{mg} \mathrm{kg}^{-1}\right)\end{array}$} \\
\hline & & & Tomate & Calabaza & Chile \\
\hline \multirow[t]{4}{*}{4} & Diazinón & $\begin{array}{l}0.011 \pm \\
0.0016\end{array}$ & 0.5 & 0.5 & ---- \\
\hline & Malatión & $\begin{array}{l}0.014 \pm \\
0.0012\end{array}$ & 3.0 & --- & 0.5 \\
\hline & Clorpirifos & $\begin{array}{l}0.002 \pm \\
0.0002\end{array}$ & --- & --- & --- \\
\hline & $\begin{array}{l}\text { Paratión } \\
\text { metílico }\end{array}$ & $\begin{array}{l}0.044 \pm \\
0.0022\end{array}$ & na & --- & na \\
\hline \multirow[t]{3}{*}{5} & Diazinón & $\begin{array}{l}0.132 \pm \\
0.0223\end{array}$ & 0.5 & 0.5 & --- \\
\hline & Clorpirifos & $\begin{array}{l}0.017 \pm \\
0.0030\end{array}$ & --- & --- & --- \\
\hline & $\begin{array}{l}\text { Paratión } \\
\text { metílico }\end{array}$ & $\begin{array}{l}0.056 \pm \\
0.0029\end{array}$ & na & --- & na \\
\hline \multirow[t]{3}{*}{6} & Diazinón & $\begin{array}{l}0.060 \pm \\
0.0075\end{array}$ & 0.5 & 0.5 & --- \\
\hline & Clorpirifos & $\begin{array}{l}0.005 \pm \\
0.0002\end{array}$ & --- & --- & --- \\
\hline & $\begin{array}{l}\text { Paratión } \\
\text { metílico }\end{array}$ & $\begin{array}{l}0.013 \pm \\
0.0020\end{array}$ & na & --- & na \\
\hline \multicolumn{6}{|c|}{$\begin{array}{l}{ }^{\dagger}=\text { Se consideraron únicamente campos con muestras positivas. }{ }^{\dagger \dagger}=\text { Los } \\
\text { valores de concentración son promedios de cuatro repeticiones por cam- } \\
\text { po. } \dagger^{\dagger \dagger}=\text { Autorizado por Codex Alimentarius }(1999) \text {. --- = No se en- }- \text { so autorizado su } \\
\text { cuentra en el listado aprobado para esa hortaliza. na = No áto } \\
\text { uso por el Codex Alimentarius (1999). LMR = Límite máximo de resi- } \\
\text { duos. }\end{array}$} \\
\hline
\end{tabular}

En los Campos 4, 5 y 6 (Cuadro 2) se encontraron residuos de diazinón, paratión metílico y clorpirifos. En el caso de diazinón en nopal, comparado con tomate y calabaza, su concentración no excedió los LMRs para su consumo. En chile el diazinón no se encuentra en el listado de insecticidas autorizados, el paratión no está autorizado para su uso en hortalizas, y clorpirifos no se encuentran reconocidos por el Codex Alimentarius. Los valores de la ingesta diaria estimada (IDE) y su comparación con la ingesta diaria admisible (IDA), establecida por organismos internacionales, para los insecticidas analizados se muestran en el Cuadro 3. La suma de la IDE para las muestras obtenidas en $\mathrm{mg}$ por $\mathrm{kg}$ de peso corporal por día, en los Campos 4, 5 y 6 fue de $0.0014,0.0040$ y $0.0016 \mathrm{mg} \mathrm{kg}^{-1}$ respectivamente, y la suma de IDA fue de $0.33 \mathrm{mg} \mathrm{kg}^{-1}$ para el Campo 4 y de $0.035 \mathrm{mg} \mathrm{kg}^{-1}$ para los Campos $5 \mathrm{y}$ 6. Es decir, el riesgo toxicológico para la salud humana 
por insecticidas provenientes del consumo de nopal es 1000 veces menor que la dosis admisible.

Cuadro 3. Ingesta diaria admisible (IDA) con relación a la ingesta diaria estimada (IDE) de residuos encontrados en nopal fresco.

\begin{tabular}{|c|c|c|c|}
\hline Campo ${ }^{\dagger}$ & $\begin{array}{l}\text { Residuos de } \\
\text { plaguicidas } \\
\text { encontrados }\end{array}$ & $\begin{array}{c}\text { IDA } \\
\left(\mathrm{mg} \mathrm{kg}^{-1} \mathrm{~d}^{-1}\right)\end{array}$ & $\begin{array}{c}\text { IDE } \\
\left(\mathrm{mg} \mathrm{kg}^{-1} \mathrm{~d}^{-1}\right) \\
1 \times 10^{-3}\end{array}$ \\
\hline 4 & $\begin{array}{l}\text { Diazinón } \\
\text { Malatión } \\
\text { Clorpirifos } \\
\text { Paratión metílico }\end{array}$ & $\begin{array}{c}0-0.005^{\dagger \dagger} \\
0.3^{\Phi} \\
0-0.01^{\dagger \dagger} \\
0.02^{\xi}\end{array}$ & $\begin{array}{l}0.0002 \\
0.0003 \\
0.00004 \\
0.0009\end{array}$ \\
\hline 5 & $\begin{array}{l}\text { Diazinón } \\
\text { Clorpirifos } \\
\text { Paratión metílico }\end{array}$ & $\begin{array}{c}0-0.005^{\dagger \dagger} \\
0-0.01^{\dagger \dagger} \\
\quad 0.02^{\xi}\end{array}$ & $\begin{array}{l}0.0026 \\
0.0003 \\
0.0011\end{array}$ \\
\hline 6 & $\begin{array}{l}\text { Diazinón } \\
\text { Clorpirifos } \\
\text { Paratión metílico }\end{array}$ & $\begin{array}{c}0-0.005^{\dagger \dagger} \\
0-0.01^{\dagger \dagger} \\
0.02^{\xi}\end{array}$ & $\begin{array}{l}0.0012 \\
0.0001 \\
0.0003\end{array}$ \\
\hline
\end{tabular}

${ }^{\dagger}=$ Se consideraron únicamente los campos con muestras positivas. PC $=$ Peso Corporal. ${ }^{\dagger \dagger}$ Pesticide Residues in Food (2004). "Pesticide Residues in Food (2006). ${ }^{\xi}$ Kamrin M A (1997).

Calonge et al. (2002) determinó la presencia de residuos de siete insecticidas organofosforados en peras (Pyrus communis L.) y manzanas (Malus domestica Borkh) adquiridas en la provincia de León, España, y en ningún caso los valores encontrados superaron los LMRs establecidos por la legislación española. Al comparar con la IDA se estimó un amplio margen de seguridad para los insecticidas (especialmente diazinón) entre 44 y 500 veces la dosis diaria admisible, por lo que los autores concluyeron que el riesgo toxicológico por consumo de peras y manzanas de insecticidas organofosforados es mínimo, aunque mayor que en el estudio realizado con nopal. Siqueiros (1988; Com. personal $)^{2}$ detectó residuos de insecticidas en productos hortícolas de consumo frecuente (tomate, chile y calabaza), muestreados en diferentes comercios de Hermosillo, Sonora; observó que para la mayoría de los insecticidas detectados no hay aprobación para su uso en hortalizas, y al hacer las estimaciones de la IDA concluyó que en la mayoría de las muestras se encontraron residuos en concentraciones que equivalen aproximadamente a $1 \%$ de la IDA, similares a los encontrados en nopal en los Campos 4, 5 y 6 de Hermosillo. Es importante destacar que los va-

\footnotetext{
${ }^{2}$ Siqueiros G (1998) Método mejorado y su aplicación para la detección de plaguicidas en tomate (Lycopersicum esculentum) chile (Capsicum annuum) y calabaza (Cucurbita pepo). Tesis de Maestría. Centro de Investigación en Alimentación y Desarrollo (CIAD). Hermosillo, Sonora, México.
}

lores establecidos por los organismos encargados del uso y manejo de plaguicidas, así como las investigaciones toxicológicas en humanos y otros mamíferos (especialmente LMR e IDA), se estiman para residuos individuales sin hacer consideración de su acción sinérgica que ocasionaría mayor daño al presentarse en grupos (Coscolla, 1993).

No se encontraron otros estudios similares de detección de residuos de insecticidas en nopal para comparar con los resultados aquí obtenidos, ya que el número y tipo de insecticidas es muy variado y además pueden variar en comportamiento.

Por los resultados mencionados, es mínimo el riesgo toxicológico derivado del consumo de nopal tratado con los insecticidas (malatión, paratión metílico, diazinón y clorpirifos), ya que el margen de seguridad fue de 1000 veces la dosis admisible en otras verduras. Además, el método de extracción en fase dispersiva puede ser aplicado como una técnica de análisis de rutina en el monitoreo de residuos de insecticidas organofosforados en nopal fresco y en su producto deshidratado.

\section{CONCLUSIONES}

En las condiciones de este estudio, el riesgo potencial toxicológico por insecticidas provenientes del consumo del nopal fresco es mínimo, pues la ingesta diaria estimada es 1000 veces menor que la dosis admisible. En el nopal deshidratado no se detectaron residuos de plaguicidas. Los efectos tóxicos derivados de la exposición permanente a pequeñas cantidades de plaguicidas hace necesaria la optimización de las técnicas analíticas, como el método de fase-sólida dispersiva, que permitan el análisis de mayor número de muestras de forma rápida, sencilla y ecológica.

\section{AGRADECIMIENTOS}

A la Q.B. Mireya Díaz por el apoyo en la logística de los muestreos de nopal y el contacto con los productores, y a la M.C. Patricia Grajeda Cota por su apoyo técnico en la implementación del método de análisis de residuos de plaguicidas en nopal.

\section{BIBLIOGRAFÍA}

Aldana M L, C I Mendívil, C D Mada, M I Silveira, J L Navarro (2003) Alteraciones en el análisis del líquido seminal de aplicadores de insecticidas en el medio urbano. Acta Med. Son. 4:5-7.

Anastassiades M, S J Lehotay, D Stajnbaher, F J Schenck (2003) Fast and easy multiresidue method employing acetonitrile extraction/partitioning and "Dispersive Solid-Phase Extraction" for the determination of pesticide residues in produce. J. AOAC Internatl. 86:412-431. 
Berger H, A Rodríguez, L Galletti (2006) Operaciones de Campo para la Utilización Agroindustrial de los Nopales. In: Utilización Agroindustrial del Nopal. C Rosell (ed). Boletín de Servicios Agrícolas de la FAO No. 162. Roma, Italia. 24 p.

Burgos A, C O García, M L Aldana, M M Meza (2005) Detection and quantification of insecticides in shrimp grown in a coastal farm in Sonora, México. Bull. Environ. Contam. Toxicol 74:335341.

Burgos A, M G Leyva, M L Aldana, C O García, C I Mendívil, E C Rosas, R Ramírez (2006) Presence of insecticides in shrimp farms adjacent to the Sea of Cortes: detection, quantification, and toxicity testing. Eur. Food Res. Technol. 222:380-384.

Calonge M, Y Pérez Pertrejo, C Ordóñez, R M Reguera, R Balaña Fouce, D Ordóñez (2002) Determinación de residuos de siete insecticidas organofosforados en frutas mediante cromatografía de gases con detector de nitrógeno-fósforo y confirmación por espectrometría de masas. Rev. Toxicol. 19:55-60.

CICOPLAFEST (1998) Catálogo Oficial de Plaguicidas: Comisión Intersecretarial para el Control del Proceso y Uso de Plaguicidas, Fertilizantes y Sustancias Tóxicas. Catálogo Oficial de Plaguicidas. Secretaria de Agricultura, Ganadería y Desarrollo Rural. México D.F.

CIESTAAM-ASERCA (1995) Centro de Investigaciones Económicas, Sociales y Tecnológicas de la Agroindustria y la Agricultura Mundial, Apoyos y Servicios a la Comercialización Agropecuaria. Mercado Mundial del Nopalito. Chapingo, México, D.F. $18 \mathrm{p}$.

Codex Stan 185 (1993) Tropical Fresh Fruits and Vegetables. FAO/WHO. Roma, Italia. http://www.codexalimentarius.net/ $\mathrm{mrls} /$ pestdes/jsp/pest_q-s.jsp (last accessed 20 June 2007).

Codex Alimentarius (1999) Tropical Fresh Fruits and Vegetables. FAO/WHO. Roma, Italia. http://www.codexalimentarius.net/ mrls/pestdes/jsp/pest_q-s.jsp (last accessed 20 June 2007).

Coscolla R (1993) Residuos de Plaguicidas en Alimentos Vegetales. Ed. Mundi-Prensa. Madrid, España. 133 p.

Dogheim S M, S A Gad Alla, S M El-Syes, M M Almaz, E Y Salama (1996) Organochlorine and organophosphorous pesticide residues in food at Egytian local markets. J. AOAC Internatl. 79:949-952.

Dogheim S M, S A Gad Alla, A M El-Marsafy, S M Fahmy (1999) Monitoring pesticide residues in Egyptian fruits and vegetables in $1995 \mathrm{~J}$. AOAC Internatl. 82:948-955.

European Community, Brussels (1990) EC Council Directive 90/642/EFC of 27 November 1990 on the Fixing of Maximum Levels for Pesticide Residues in and on Fruit and Vegetables. Official Journal of European Communities L350,0071.

Haney P Y, C A Harris (1999) The variations of pesticide residues in fruits and vegetables and the associated assessment of risk. Regul. Toxicol. Pharmacol. 30:S34-S41.

Juran J M, F M Gryma (1997) Manual de Control de Calidad. Vol. 1, 4a ed. Mc Graw Hill. México. 34 p.

Kamrin M A (1997) Organophosphates. In: Pesticide Profiles: Toxicity, Environmental Impact, and Fate. CRC Lewis Publishers. New York, USA. 199 p.

Louvar J, D Louvar (1998) Health and Environmental Risk Analysis: Fundamental with Applications. Prentice Hall PRT, USA. 58

Meloan C E (1998) Manual de Entrenamiento para Laboratorio de Pesticidas. J. AOAC Internatl. USA. $155 \mathrm{p}$.

Montenegro R A (2001) Informe Sobre los Riesgos Sanitarios y Ambientales del Malatión. http://webs.chasque.net/ rapaluy /malation/informe2.html (last accessed 20 June 2004).
Navarro S, M A Cámara, A Barba, R Toledano, A Luna (1992) Incident of residual levels of organophosphate insecticides in faro produce in the Region of Murcia, Spain comparison of intake in the 1985-1986 and 1989 campaigns. J. Appl. Toxicol. $12: 251-254$

Pesticide Residues in Food (2004) Report of the Joint FAO Panel of Experts on Pesticide Residue in Food and the WHO Core Assessment Group. http://whqlibdoc.who.int/publications/ 2005/ 9251052425.pdf (last accessed 06 February 2008).

Pesticide Residues in Food (2006) Report of the Joint FAO/WHO Meeting on Pesticide Residues. http://www.fao.org/ag/AGP/ AGPP/pesticid/JMPR/DOWNLOAD/2006 (last accessed 06 February 2008).

Platt C, A Montoy, L López (2003) Optimización en el Proceso de Deshidratación de Nopal (Opuntia ficus -indica) Utilizando una Combinación de Secadores. Mem. IX Congreso Nacl y VII Internacional sobre el Cultivo y Aprovechamiento del Nopal. G Esparza, Salas M A, J Mena, D R Valdez (eds). Zacatecas, Zac., 2 al 6 de Septiembre, 2003. Universidad Autónoma de Chapingo, Estado de México, México. pp:236-241.

Reigart R J, R James (1999) Reconocimiento y Manejo de los Envenenamientos por Pesticidas. 5a ed. http://www.epa.gov/ pesticides/safety/healthcare (last accessed 08 January 2008).

Ripley B, G Ritcey, R Harris, M A Denommé, P Brown (2001) Pyrethroid Insecticide Residues on Vegetable Crops. Pest Manage. Sci. 57:683-687.

Torres C M, Y Pico, J Mañes (1996) Determination of Pesticide Residues in Fruit and Vegetables. J. Chromat. A 754:301-331.

SIAP-SAGARPA (2007) Sistema de Información Agroalimentaria y Pesquera - Secretaría de Agricultura, Ganadería, Desarrollo $\mathrm{Ru}-$ ral, Pesca y Alimentación. Anuarios Estadísticos. www.siap.sagarpa.gob.mx/ar_comanuar.html (last accessed 19 August 2007).

USDA, United States Department of Agriculture (1991) Analytica Chemistry Laboratory Guidebook. Residue Chemistry. Science and Technology. USDA.FSIS. Washington, D.C., USA.

Valenzuela A I, Y Picó, G Font (2001) Monitoring of five pesticide residues in oranges by matrix solid phase dispersion and liquid chromatography: estimation of daily intake. J. AOAC Internatl. 84:900-909.

Valenzuela A I, R Armenta, E Moreno, L Gutiérrez, P Grajeda, C Orantes (2006) Optimización y validación de un método de dispersión de matriz en fase sólida para la extracción de plaguicidas organofosforados en hortalizas. Rev. Fac. Agron. (LUZ) 23:464-474.

Valdez S, M L Aldana, M I Silveira, G Rodríguez,F J Wong, J Borboa (2002) Monitoreo de residuos de plaguicidas en el grano de trigo y sus fracciones producido y almacenado en Sonora. Mem. del IV Congreso del Noroeste en Ciencias Alimentarias y Biotecnología. Hermosillo, Son. 13 al 16 de Noviembre, 2002. Universidad de Sonora, México. pp:1-18.

Vargas N D, M L Aldana, M I Silveira, G Rodríguez, F J Wong, J Borboa (2002) Identificación y cuantificación de residuos de insecticidas en granos de maíz, frijol y garbanzo en el Estado de Sonora, mediante la técnica de cromatografía de gases. Mem. del IV Congreso del Noroeste en Ciencias Alimentarias y Biotecnología Hermosillo, Son. 13 al 16 de Noviembre, 2002. Universidad de Sonora, México. pp:1-15

Vázquez M L, A Langure, C Orantes, M E Flores, M C Bermúdez (1999) Incidence of pesticide residues in adiposities tissue of beef, pork and poultry from plants located in northwestern Mexico. J. Muscle Foods 10:295-303. 